\title{
High-Speed Optical Coherence Tomography System Using a 200-kHz Swept Light Source with a KTN Deflector
}

\author{
Yuichi Okabe $^{1,2}$, Yuzo Sasaki ${ }^{1}$, Masahiro Ueno ${ }^{1}$, Takashi Sakamoto ${ }^{1}$, Seiji Toyoda ${ }^{1}$, \\ Junya Kobayashi ${ }^{1}$, Masato $\mathrm{Ohmi}^{2}$ \\ ${ }^{1}$ NTT Photonics Laboratories, Nippon Telegraph and Telephone Corporation, Kanagawa, Japan \\ ${ }^{2}$ Graduate School of Medicine, Course of Allied Health Science, Osaka University, Osaka, Japan \\ Email: okabe.yuichi@lab.ntt.co.jp
}

Received March 7, 2013; revised April 9, 2013; accepted April 17, 2013

Copyright (C) 2013 Yuichi Okabe et al. This is an open access article distributed under the Creative Commons Attribution License, which permits unrestricted use, distribution, and reproduction in any medium, provided the original work is properly cited.

\begin{abstract}
We demonstrate a high-speed swept-source optical coherence tomography (SS-OCT) system, which is based on a highspeed swept light source and a Mach-Zehnder interferometer with a high-speed photodetector. The light source is an external-cavity laser tuned by a KTN electro-optic deflector, which exhibits a very fast response and large deflection. The scanning wavelength range is almost $80 \mathrm{~nm}$ up to $200 \mathrm{kHz}$ with a $\pm 400-\mathrm{V}$ deflector driving voltage. The system acquires $1 \mathrm{~mm} \times 1 \mathrm{~mm}$ images consisting of $200 \times 200$ pixels within few milliseconds. We present preliminary SS-OCT images of an in-vitro human nail and an in-vivo finger pad.
\end{abstract}

Keywords: Swept-Source Optical Coherence Tomography; KTN; New Light Source

\section{Introduction}

Optical coherence tomography using a swept light source (SS-OCT), a laser interferometry technique that can acquire cross-sectional images of tissue and other scattering materials with micrometer-resolution, is attractive for biomedical applications [1,2]. SS-OCT has potential for high-speed data acquisition among the variations of OCT. Motion artifacts caused by bodily movements, such as pulsation, internal organs motion, and endoscopic motion, decrease the quality of in-vivo OCT images. SS-OCT characteristics, as a high-speed data acquisition and a rapid image rendering, can reduce undesirable effects arising from these motion artifacts [2,3].

SS-OCT detects wavelength-resolved interference fringes generated by a wavelength scanning laser. A key component of an SS-OCT system is the light source. The desirable output characteristics of a swept light source include high power, a high repetition rate, a narrow instantaneous linewidth, and a broad sweep wavelength range $[2,3]$. As the repetition rate of the light sources is increased, the duration of the laser irradiation decreases and the quantity of data obtained within a fixed measurement duration increases [4]. Thus far, such light sources have used mechanical actuators for wavelength scanning $[5,6]$. In contrast, we are developing a new light source that incorporates an electro-optic deflector and using it in a high-speed SS-OCT system. The deflector is based on a $\mathrm{KTa}_{1-\mathrm{x}} \mathrm{Nb}_{\mathrm{x}} \mathrm{O}_{3}(\mathrm{KTN})$ single crystal. KTN has a very large electro-optic effect, which changes a refractive index by an applied voltage and bends the path of a light beam in a direction $[7,8]$. The deflection effect of the KTN is caused by a non-uniform electric field generated by injected carriers, and the deflector exhibits a fast response and fairly large light deflection angle $[9,10]$. The KTN deflector is useful as a fast repetition rate swept source.

Taking the advantages of the swept light source using the KTN deflector into consideration, with the aim of advanced image rendering while minimizing the blur caused by bodily movement, we are developing a highspeed SS-OCT system using the light source. Preliminary OCT images of a human nail and finger pad are presented.

\section{Swept Light Source with KTN Deflector}

Figure 1 shows our light source with a KTN deflector, which is based on a Littman-Metcalf cavity configuration [11]. The cavity consists of a $1.3-\mu \mathrm{m}$ semiconductor optical amplifier (SOA) module, a collimator, a $1.5 \mathrm{~mm}$ thick KTN deflector with electrodes, and a cylindrical concave lens with a focal length of $-12.7 \mathrm{~mm}$, a grazingincident $600 \mathrm{l} / \mathrm{mm}$ grating, and a high reflector (HR). The 


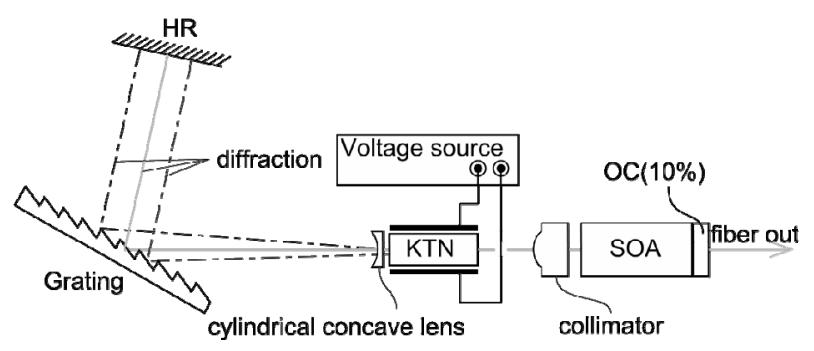

Figure 1. Setup of a swept light source equipped with a KTN deflector. HR: high reflector. SOA: semiconductor optical amplifier. OC: output coupler.

SOA module has a $10 \%$ reflector coupled to an optical fiber through an optical isolator on one side. The cavity length between the $10 \%$ reflector and the HR is about 60 $\mathrm{mm}$.

The KTN deflector operates by exploiting trapped electrons in the KTN crystal. The electrons are injected and kept trapped by supplying a DC voltage to the KTN (called "pre-charging") prior to the application of a highfrequency AC voltage for scanning. KTN can follow the driving voltage faster than that allowed by electron mobility. However, KTN simultaneously exhibits the characteristics of a cylindrical convex lens because of the trapped electrons [9]. We compensate for the convex lens with a cylindrical concave lens, because the lens power inside the cavity degrades the instantaneous linewidth of the laser. A light emitted from the SOA is collimated with the collimator and reaches the HR through the KTN deflector and the grating. The filter is constructed using the KTN deflector, the grating, and the HR. Only light of certain wavelength is selected according to a deflection angle of the KTN deflector and returned to the SOA.

We pre-charged the KTN crystal by applying \pm 500 -V DC for ten seconds and then scanned the laser wavelength by applying a $\pm 400-\mathrm{V}$ sinusoidal voltage to the crystal.

Figure 2 shows a typical integrated spectrum of the light source. The scanning range remained constant at 80 $\mathrm{nm}$ up to a repetition rate of $200 \mathrm{kHz}$.

Figure 3 shows the $200-\mathrm{kHz}$ voltage applied to the KTN deflector and the corresponding interference fringe signal. The repetition rate is currently limited by our particular high-voltage driver. The KTN deflector has been shown to have a flat response up to $400 \mathrm{kHz}$ when operated after pre-charging $[9,10]$.

Figure 4 shows point-spread functions (PSFs), namely, the relative OCT signal intensity for a single layer reflection at various optical delays between the sample and reference arms.

The PSFs are obtained by Fourier transforming the interference fringe signals. We observed a decrease in the interferometry fringe amplitude [or the peak height of its fast Fourier transform (FFT)] as we increased the optical

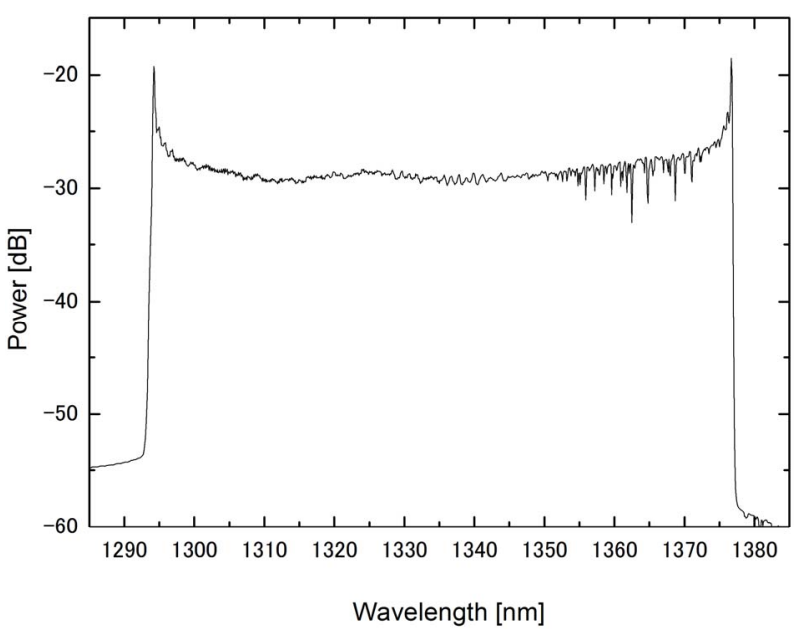

Figure 2. Measured integrated spectrum when the swept light source is scanned sinusoidally at $200 \mathrm{kHz}$.

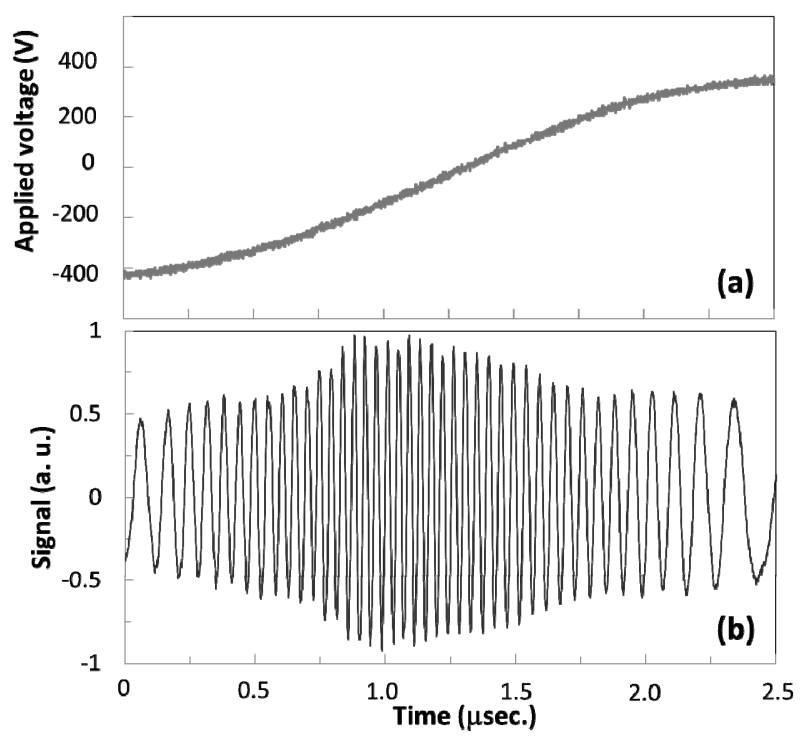

Figure 3. (a) Voltage applied to the KTN deflector and (b) corresponding interference fringe signal.

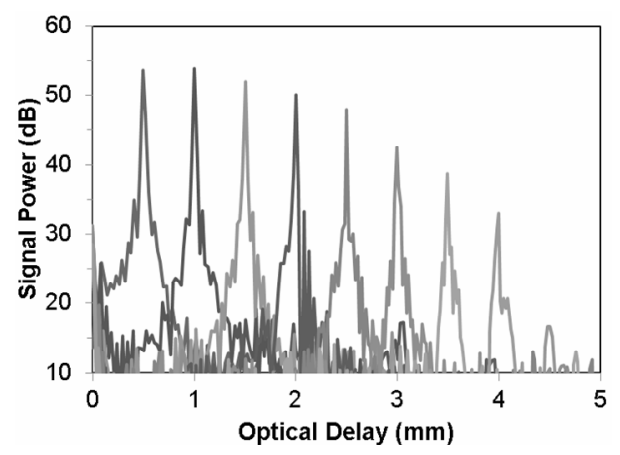

Figure 4. Measured PSFs at $200 \mathrm{kHz}$ scan.

delay. The measured coherence length in air was approximately $6 \mathrm{~mm}$ for a $200-\mathrm{kHz}$ scan. This indicates that a depth range of several millimeters can be covered 
using this frequency swept light source.

\section{High-Speed SS-OCT System Setup}

The entire system is shown in Figure 5. The setup consists of a swept light source with a KTN deflector, an optical fiber interferometer with a Mach-Zehnder configuration, a galvano scanning mirror, and a computer for signal processing. The source outputs light at $20 \mathrm{~mW}$ around a center wavelength of $1.33 \mu \mathrm{m}$, with a wavelength scanning range of $80 \mathrm{~nm}$. Light from the source is split by a 10/90 coupler into a reference arm and a sample arm. The lights reflected from the reference mirror and the samples are separated by respective optical circulators. Polarization controllers in both arms are used to adjust the polarization states of the two arms. Light through the two arms interferes at a 50/50 coupler, the outputs of which are detected by a balanced photodetector.

Interference fringe signals are recorded on a channel of a 10-bit data acquisition board at a sampling rate of 1 GSample/s. A depth profile, as a single A-line scan, is obtained by the FFT of the interference fringe signal. A 2D image is constructed by mapping a transversal set of A-line scans by operating the galvano scanning mirrors (B-line scan).

\section{OCT Image Results}

As preliminary measurements, we acquired human tissue images using the high-speed SS-OCT system. The $200 \times$ 200 pixel images covered a sample area of approximately $1 \mathrm{~mm} \times 1 \mathrm{~mm}$. The image acquisition time was few milliseconds, which is several times faster than conventional SS-OCT systems.

Figure 6(a) shows a typical in-vitro OCT image of a human nail, which is about $0.5-\mathrm{mm}$-thick. Here the top and bottom of the nail surfaces are clearly visualized as bright lines.

Figure 7(a) is an in-vivo image of a human finger pad.

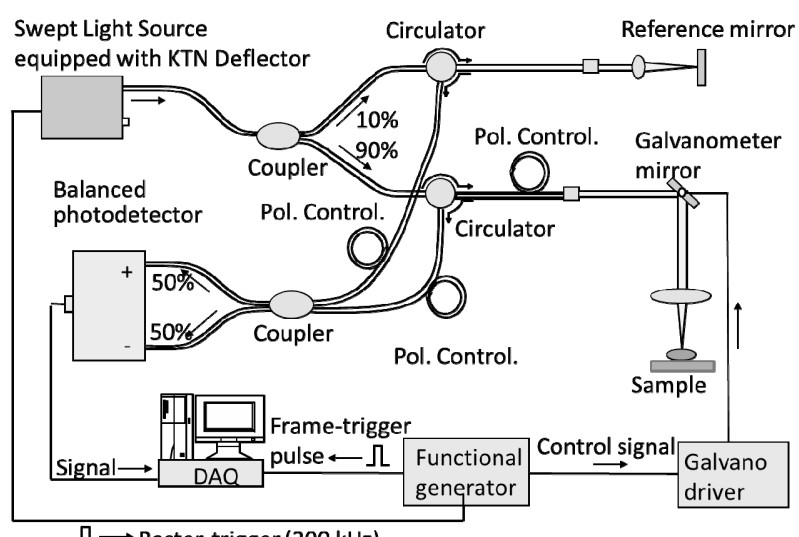

$\Omega \longrightarrow$ Raster-trigger $(200 \mathrm{kHz})$

Figure 5. Setup of high-speed SS-OCT system.
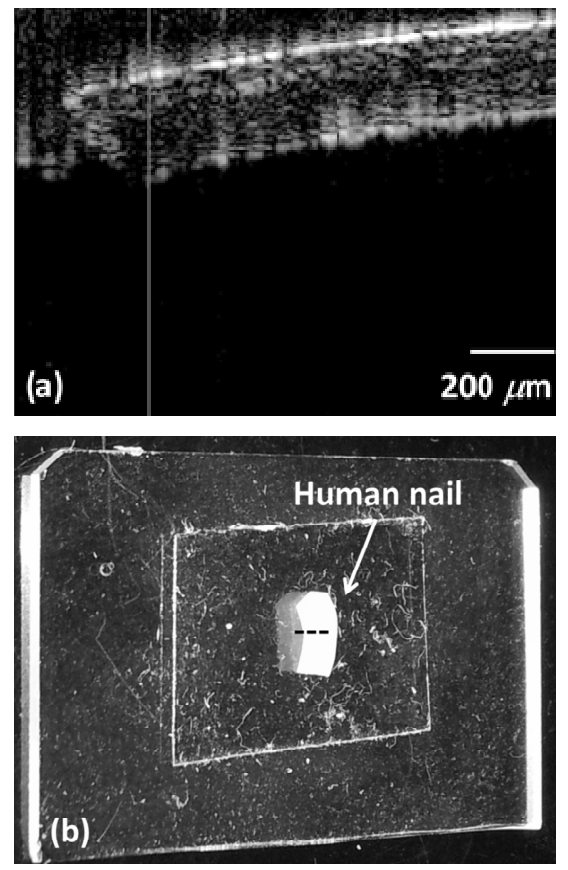

Figure 6. (a) In-vitro OCT image of a human nail; (b) Photograph of the human nail showing the scan line.
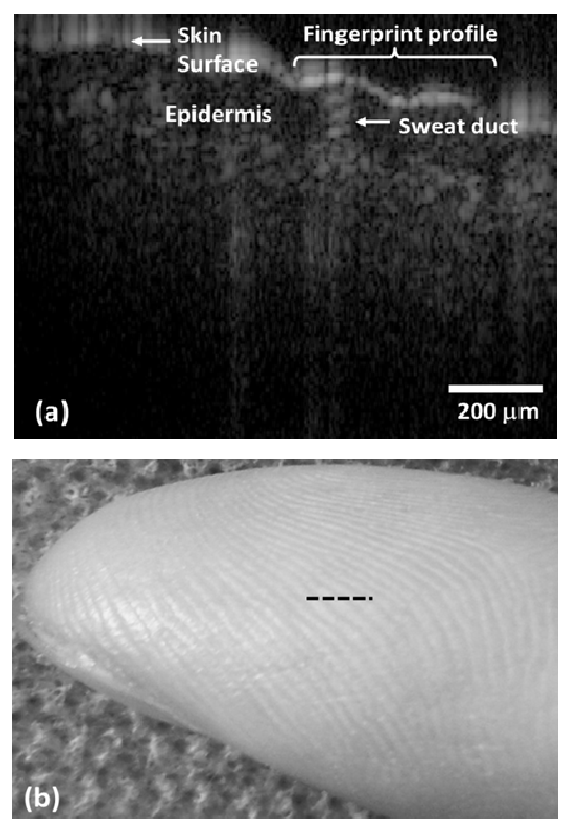

Figure 7. (a) In-vivo OCT image of a human finger pad; (b) Photograph of the human finger pad showing the scan line.

The skin surface, sweat duct, and epidermis can be seen in the image. The sweat duct appears as a spiral feature. The contrast, resolution, and penetration depth of the images will be improved by additional signal processing, which includes recalibrating and resampling to a uniform k-space before image construction. A detailed evaluation of the resolution after such signal processing will be discussed in future work. 


\section{Conclusions}

We proposed and demonstrated a high-speed SS-OCT system, which is based on a high-speed swept light source and a Mach-Zehnder interferometer with a highspeed balanced photodetector. The light source is an external-cavity laser tuned by a KTN electro-optic deflector, which exhibits a very fast response and large deflection.

The in-vitro measurement of a human nail and in-vivo measurement of a human finger pad were presented as examples of OCT images obtained at an A-line rate of $200 \mathrm{kHz}$. The $200 \times 200$ pixel images covered a sample area of approximately $1 \mathrm{~mm} \times 1 \mathrm{~mm}$.

Our light source, which is a key component of SSOCT, has the potential to achieve a higher repetition rate. The KTN deflector operation up to $400 \mathrm{kHz}$ has been reported $[9,10]$. Moreover, KTN itself has a $500-\mathrm{MHz}$ EO response [12]. Hence, the further improvement of the current system performance can be expected. We believe that such a system has significant potential for biomedical cross-sectional imaging applications where highspeed measurements are crucial.

\section{REFERENCES}

[1] S. R. Chinn, E. A. Swanson and J. G. Fujimoto, "Optical Coherence Tomography Using a Frequency-Tunable Optical Source," Optics Letters, Vol. 22, No. 5, 1997, pp. 340-342. doi:10.1364/OL.22.000340

[2] S. H. Yun, G. J. Tearney, J. F. de Boer, N. Iftimia and B. E. Bouma, "High-Speed Optical Frequency-Domain Imaging," Optics Express, Vol. 11, No. 22, 2003, pp. 29532963. doi:10.1364/OE.11.002953

[3] W. Y. Oh, S. H. Yun, G. J. Tearney and B. E. Bouma, "Wide Tuning Range Wavelength-Swept Laser with Two Semiconductor Optical Amplifiers," IEEE Photonics Technology Letters, Vol. 17, No. 3, 2005, pp. 678-680. doi:10.1109/LPT.2004.841003

[4] R. Huber, D. C. Adler, V. J. Srinivasan and J. G. Fujimoto, "Fourier Domain Mode Locking at $1050 \mathrm{~nm}$ for Ultra-High-Speed Optical Coherence Tomography of the Human Retina at 236000 Axial Scans per Second," Op- tics Letters, Vol. 32, No. 14, 2007, pp. 2049-2051. doi:10.1364/OL.32.002049

[5] R. Huber, M. Wojtkowski and J. G. Fujimoto, "Fourier Domain Mode Locking (FDML): A New Laser Operating Regime and Applications for Optical Co-Herence Tomography," Optics Express, Vol. 14, No. 8, 2006, pp. 3225 3237. doi:10.1364/OE.14.003225

[6] M. Kuznetsov, W. Atia, B. Johnson and D. Flanders, "Compact Ultrafast Reflective Fabry-Perot Tunable Lasers for OCT Imaging Applications," SPIE Proceedings, Vol. 7554, 2010, Article ID: 75541F. doi:10.1117/12.842567

[7] K. Nakamura, J. Miyazu, M. Sasaura and K. Fujiura, "Wide-Angle, Low-Voltage Electro-Optic Beam Deflection Based on Space-Charge-Controlled Mode of Electrical Conduction in $\mathrm{KTa}_{1-\mathrm{x}} \mathrm{Nb}_{\mathrm{x}} \mathrm{O}_{3}$," Applied Physics Letters, Vol. 89, No. 13, 2006, Article ID: 131115. doi:10.1063/1.2357335

[8] T. Itoh, M. Sasaura, S. Toyoda, K. Manabe, K. Nakamura and K. Fujiura, "High-Frequency Response of ElectroOptic Single Crystal $\mathrm{KTa}_{1-\mathrm{x}} \mathrm{Nb}_{\mathrm{x}} \mathrm{O}_{3}$ in Paraelectric Phase," Conference on Lasers and Electro-Optics, Optical Society of America, 2005.

[9] J. Miyazu, Y. Sasaki, K. Naganuma, T. Imai, S. Toyoda, T. Yanagawa, M. Sasaura, S. Yagi and K. Fujiura, "400 $\mathrm{kHz}$ Beam Scanning Using $\mathrm{KTa}_{1-\mathrm{x}} \mathrm{Nb}_{\mathrm{x}} \mathrm{O}_{3}$ Crystals," Proceedings of CLEO, San Jose, 16-21 May 2010, pp. 1-2.

[10] J. Miyazu, T. Imai, S. Toyoda, M. Sasaura, S. Yagi, K. Kato, Y. Sasaki and K. Fujiura, "New Beam Scanning Model for High-Speed Operation Using $\mathrm{KTa}_{1-\mathrm{x}} \mathrm{Nb}_{\mathrm{x}} \mathrm{O}_{3}$ Crystals," Applied Physics Express, Vol. 4, No. 11, 2011, Article ID: 111501. doi:10.1143/APEX.4.111501

[11] Y. Okabe, Y. Sasaki., M. Ueno, T. Sakamoto, S. Toyoda, S. Yagi, K. Naganuma, K. Fujiura, Y. Sakai, J. Kobayashi, K. Omiya, M. Ohmi and M. Haruna, "200 kHz Swept Light Source Equipped with KTN Deflector for Optical Coherence Tomography," Electronics Letters, Vol. 48, No. 4, 2012, pp. 201-202. doi:10.1049/el.2011.4057

[12] S. Toyoda, K. Fujiura, M. Sasaura, K. Enbutsu, A. Tate, M. Shimokozono, H. Fushimi, T. Imai, K. Manabe, T. Matsuura and T. Kurihara, "Low-Driving-Voltage Electro-Optic Modulator with Novel $\mathrm{KTa}_{1-\mathrm{x}} \mathrm{Nb}_{\mathrm{x}} \mathrm{O}_{3}$ Crystal Waveguides," Japanese Journal of Applied Physics, Vol. 43, 2004, pp. 5862-5866. doi:10.1143/JJAP.43.5862 